\title{
EL ACOSO EN LA ADMINISTRACIÓN PÚBLICA. UNA PROPUESTA DE INTERVENCIÓN A TRAVÉS DE LA MEDIACIÓN
}

\author{
Labor Bullying in the Public Administration. \\ A Proposal of Intervention Through Mediation
}

ESTHER GÓMEZ JiMÉNEZ

Mediadora Civil y Mercantil

\section{ABSTRACT}

Este artículo pretende analizar partiendo del concepto de acoso laboral, su incidencia en el personal de las Administraciones públicas. Para ello, en primer lugar, se contextualizará el término acoso desde diferentes perspectivas, poniendo el foco de atención en la identificación de una serie de conductas que determinan los comportamientos más frecuentes. Bajo este paraguas se plantean una serie de interrogantes, asi como la posibilidad de adoptar técnicas de resolución de conflictos, cómo es el caso de la mediación. Posteriormente, el estudio de protocolos de actuación y prevención frente al acoso laboral darán paso a una serie de conclusiones.

Palabras clave: Acoso laboral, mobbing, Administración pública, conflicto, mediación.

This article intends to analyse the concept of labour bullying and its incidence in the personnel of the public administrations. For this purpose, in the first place, we will establish the term mobbing from diverse perspectives, putting the focus of attention in the identification of a series of behaviours that determine the most frequent 
patterns of mobbing. Under this umbrella we pose a series of queries, as well as the possibility to adopt resolution techniques for conflicts, such as the case of mediation. Later, we examine the protocols of response and prevention when facing labour bullying giving pace to a series of conclusions.

Keywords: Labour bullying, mobbing, public Administration, conflict, mediation. 


\section{SUMARIO}

Sumario: 1. Introducción. 2. Contextualizando el acoso laboral. 3. Génesis del conflicto. 4. Fases del acoso y su tipología. 5. El conflicto en las Administraciones Públicas: su determinación y efectos. 6. La mediación como herramienta para la resolución de conflictos: especial referencia a protocolos de actuación y prevención. 7. Conclusiones. 8. Bibliografía.

\section{Introducción}

La actualidad del mercado de trabajo caracterizado por una alta vulnerabilidad, competitividad, y variabilidad, ponen de manifiesto el hecho de que las organizaciones se vuelvan cada vez más competitivas y entiendan que es necesario renovarse. Fruto de ello, es la importancia que ha ido cobrando el uso de determinadas técnicas de formación de personal adoptadas en el ámbito del sector privado. Cada vez son más las organizaciones privadas que apuestan por la mejora de las capacidades de su personal, lo que se traduce en una mejora de sus resultados, y por tanto del clima laboral. Por el contrario, en el sector público la adopción de estas técnicas en cuanto a mejora de las capacidades se encuentra en una fase incipiente. La Administración pública se sigue caracterizando por una cultura organizacional predominantemente rígida, que en muchas ocasiones hace que la relación laboral se vea mermada. Por un lado, porque los trabajadores se ven abocados a soportar cargas de trabajo excesivas, y por otro, porque al no saber gestionarlas, se presentan episodios de estrés, desembocando en situaciones conflictivas que repercuten en el correcto desempeño de la actividad laboral.

Por lo anteriormente mencionado, se hace indispensable la adopción de técnicas y herramientas que propicien un entorno de trabajo saludable, y que sienten las bases de una cultura de resolución de conflictos. Tal y como apunta Arciniega (2012: 88)

La cultura organizacional y determinados estilos de liderazgo están siendo cada vez más identificados como factores clave en el desarrollo del acoso laboral, a lo que añade que no se trata de un problema episódico o interpersonal, sino de un problema estructural y estratégico que tiene sus raíces en factores organizativos, culturales y sociales más amplios. 
Desde la antigüedad las Administraciones públicas se han caracterizado por una serie de factores que son identificados por Goiría et.al. (2007: 42)

— Excesiva rigidez de la organización que provoca un sistema cerrado, incapaz de adaptarse a las situaciones cambiantes.

- Dificultad de movilidad de los trabajadores que trabajan a kilómetros de sus domicilios, lo que dificulta la conciliación de la vida familiar y laboral, produciendo estrés y aumento de conflictos.

— Dificultad para llevar a cabo los procedimientos, el desempeño requiere más rapidez y agilidad por lo que se llevan a cabo soluciones que pueden ser arbitrarias.

Las Administraciones públicas deben evolucionar hacia un modelo de trabajo basado en la participación, equidad, y motivación de los empleados transformando su modelo de gestión en un modelo más comunicativo y eficaz.

Dado que actualmente, cada vez son más las organizaciones que deciden adoptar mecanismos de resolución de conflictos, ya sea mediante la adopción de protocolos, códigos de buenas conductas o con la formación de personal especializado, resulta de especial relevancia poner el foco de atención en la búsqueda y adopción de herramientas para la resolución de los conflictos, como es el caso de la mediación. Para García Sánchez (2007: 50)

El hecho de que los trabajadores o recursos humanos del sector público constituyan el principal input en la prestación de servicios, otorga una significativa importancia a la gestión de los mismos, constituyendo el factor de mayor valor estratégico con el que cuentan las administraciones para adaptarse al cambio. Esto hace que se propicie la adopción de nuevas técnicas en la gestión de recursos humanos que permitan potenciar las capacidades de los trabajadores, a través de la desaparición de la rigidez del tradicional modelo público.

Por tanto, la rigidez de la Administración pública ligado a cargas de trabajo excesivas, es el resultado de la proliferación de situaciones de acoso en el entorno laboral. De manera que, es necesario poner el acento en la exposición de motivos que dan origen a conflictos en las Administraciones públicas, es decir intentar dar respuestas a una serie de interrogantes ¿por qué surge el acoso en la Administración pública?, ¿por qué son acosados los funcionarios? Para poder llegar así, a vislumbrar la adopción de mecanismos que frenen este tipo de conductas. En muchas ocasiones, la organización contempla la mediación, y por ello, decide formar a una serie de empleados en técnicas de resolución de conflictos. Así, mediante el conocimiento de la misma, estos empleados se convierten en mediadores y por tanto en catalizadores del conflicto. 


\section{Conceptualizando el acoso laboral}

Son muchos los términos que se utilizan para hacer referencia al acoso laboral. Se utilizan diferentes expresiones inglesas como mobbing, bullying, bossing, siendo la más conocida el término mobbing. La palabra «mob» ha sido traducida como multitud y «to mob» como acoso, acosar en masa a alguien. El término en español ha adquirido varias acepciones entre las que se encuentran el término acoso psicológico, psicoterror laboral, hostigamiento, etc.

En cuanto al concepto, son muchos los enfoques que toma dicho término, pudiendo valorarse desde varios ámbitos.

La Organización Internacional del Trabajo ha definido el acoso laboral como "la acción verbal o psicológica de indole sistemática, repetida o persistente por la que, en el lugar de trabajo o en conexión con el trabajo, una persona o un grupo de personas hiere a una victima, la humilla, ofende o amedrenta» (OIT 2002) ${ }^{1}$.

Desde el área de la psicología Leyman (1996: 27)

Lo define como aquel comportamiento que consiste en actitudes hostiles, frecuentes y repetidas en el lugar de trabajo que tienen a la misma persona en la punta de mira. Se trata, por consiguiente, de un proceso de destrucción, integrado por comportamientos hostiles que, observados aisladamente, podrían parecer anodinos pero cuya repetición constante tiene efectos perniciosos.

Seguidamente Piñuel (2007: 19) añade que:

Se trata de un acoso psicológico continuado y deliberado maltrato verbal o modal que recibe un trabajador de otro u otros, con vistas a reducirlo, someterlo, apocarlo, destruirlo psicológicamente, y que consiste en comportamientos de hostigamientos frecuentes, recurrentes y sistemáticos contra él.

Desde el enfoque sociológico se considera que el fenómeno siempre ha existido (Arciniega, 2012), a lo que Molina (2001) ańade que el mobbing es una palabra que desgraciadamente está muy de actualidad, incluso podría decirse que es un término de moda.

Desde el ámbito del derecho, el Código penal en el art. 173.1 se desprende que

el que infligiera a otra persona un trato degradante, menoscabando gravemente su integridad moral, será castigado con la pena de prisión de seis meses a dos años. Con la misma pena serán castigados los que, en el ámbito de cualquier relación laboral o

\footnotetext{
${ }^{1}$ Informe OIT, Reunión de Expertos recomendaciones prácticas sobre la violencia y el estrés en el sector servicios
} 
funcionarial y prevaliéndose de su relación de superioridad, realicen contra otro de forma reiterada actos hostiles o humillantes que, sin llegar a constituir trato degradante, supongan grave acoso contra la víctima.

\section{Para González (2009: 92)}

se trata de una situación de conflicto derivada del ejercicio ilegítimo de un poder conformador o domesticador que se lleva a cabo, de un modo continuado y sistemático, a veces también con desviación de poder, por un sujeto activo (acosador) con el propósito de domeñar el alma de un sujeto pasivo (acosado) y que tiene lugar en el seno de una organización a los que ambos pertenecen, conflicto que puede llegar a ser de tal intensidad que hace extremadamente difícil al acosado, seguir desempeñando sus funciones.

De todas las definiciones anteriormente mencionadas, se extrae que el hecho debe ser continuado y que tiene lugar durante la relación laboral. Todas estas definiciones anteriormente mencionadas desembocan en una serie de características que son identificadas por Rayner y Cooper (1997: 212) agrupándolas en:

- Amenazas del status profesional.

- Amenazas a la reputación personal.

- Aislamiento.

- Carga excesiva de trabajo.

- Desestabilización.

Para Leyman (1996:7) el terror psicológico o mobbing en la vida laboral implica una comunicación hostil e inmoral. Se trata de una situación de conflicto en el que la víctima se encuentra en situación de desamparo e indefensión.

\section{Génesis del conflicto}

En cualquier organización ya sea pública o privada la existencia de conflictos es evidente, ya que éstos surgen en distintos entornos o contextos, siendo considerados inherentes a la naturaleza humana. Al referirnos al entorno laboral, es preciso conocer las causas que los originan dando como resultado, situaciones de acoso. Para ello, se hace imprescindible puntualizar y por tanto delimitar su concepto.

Para Muñoz (2003: 98) el conflicto se presenta cuando dos actores están en situación de oposición incompatible, lo que conlleva a un enfrentamiento o lucha. Sin embargo, Touzard (1981: 24) lo define como una situación en que unos actores persiguen metas diferentes o defienden valores contradictorios, o bien persiguen de forma competitiva la misma meta. De estas definiciones se establece que para hablar de conflicto debe haber al menos dos individuos y que 
se perciba una situación de incompatibilidad de intereses. Haciendo alusión al origen del mismo, el conflicto es entendido a través de la conflictología, según Vinyamata (2003: 323)

Disciplina que ha de ser capaz de educar en los aspectos característicos de la transformación, con la finalidad de que las partes puedan regular sus conflictos por ellas mismas, haciendo uso de su empoderamiento, y con el objetivo último de reconstruir las relaciones humanas por medio de la reconciliación.

La conflictología, como sostiene Muñoz (2003: 101)

No implica actuar con presión o violencia, sino más bien examinar los déficits o insatisfacción de alguna necesidad, estableciendo diferencia entre los conflictos de intereses en los que las partes intentan hacer primar sus privilegios y ventajas sobre la otra parte.

Tal y como apunta Quera (2003: 140) la cooperación es la clave, se necesitan colaboradores para alcanzar soluciones "viables, creativas y válidas», dado que son las personas las que tienen la facultad de regular sus conflictos, siendo la vía más importante, el diálogo. A continuación, se detallan los tipos de conflictos según sus causas, atendiendo a la clasificación dada por Moore (1995: 62)

— Conflictos de relación: Se deben a fuertes emociones negativas, percepciones falsas o estereotipos, a escasa o nula comunicación, o a conductas negativas repetitivas.

- Conflictos de información: Se dan cuando a las personas les falta la información necesaria para tomar decisiones correctas, estás mal informadas, difieren sobre qué información es relevante, o tienen criterios de estimación discrepantes.

— Conflictos de intereses: Están causados por la competición entre necesidades incompatibles o percibidas como tales. Los conflictos de intereses resultan cuando una o más partes creen que para satisfacer sus necesidades, deben ser sacrificadas las de un oponente. Los conflictos fundamentados en intereses (dinero, recursos físicos, tiempo, etc.), o de procedimiento, o psicológicos (percepciones de confianza, respeto, etc.).

- Conflictos estructurales: Son causados por estructuras opresivas de relaciones humanas. Estas estructuras están configuradas muchas veces por fuerzas externas a la gente en conflicto. Escasez de recursos físicos o autoridad, condicionamientos geográficos (distancia o proximidad), tiempo (demasiado o demasiado poco), estructuras organizativas, etc.

- Conflictos de valores: Son causados por sistemas de creencias incompatibles. Los valores son creencias que la gente emplea para dar sentido a sus vidas. Las disputas de valores surgen solamente cuando unos intentan imponer por la fuerza un conjunto de valores a otros. 
En relación a los actores implicados, se clasifican de la siguiente forma:

- Conflicto intrapersonal o intrapsíquico: El origen de los conflictos incluye ideas, pensamientos, emociones, valores, predisposiciones, impulsos, que entran en colisión unos con otros.

- Conflicto interpersonal: Este tipo de conflictos ocurre entre las personas individuales, ejemplo, jefe y subordinado, amigos, etc. La mayoría de la teoría sobre negociación y mediación se refiere a la resolución de los conflictos interpersonales.

- Conflicto intragrupal: Este tipo de conflicto se da dentro de un pequeño grupo: dentro de las familias, corporaciones, clases, etc. En este nivel se analiza como el conflicto afecta a la capacidad del grupo para resolver sus disputas.

- Conflicto intergrupal: En éste último nivel el conflicto se produce entre dos grupos: ejemplo, sindicatos y patronal, etc. En este nivel el conflicto es muy complicado debido a la gran cantidad de gente implicada y a las interacciones entre ellos.

De todos esos conflictos los más notorios en la Administración pública son los conflictos interpersonales y los estructurales.

\section{Fases del acoso laboral y su tipología}

Leyman (1996) identificó cuatro fases que dan origen a situaciones de acoso laboral:

Fase 1: De conflicto: en esta fase se da la existencia de problemas puntuales que por una lado pueden solucionarse a través del diálogo o por el contrario pueden ocasionar un problema más profundo, si es así, se da paso a la siguiente fase.

Fase 2: De Mobbing o Estigmatización: En esta fase aparece el hostigamiento hacía la victima de manera sistemática, prolongada. En este momento se puede hablar de acoso psicológico.

Fase 3: De intervención de la organización: el conflicto trasciende a la organización. Se plantean dos situaciones: por un lado el conflicto puede ser resuelto positivamente por medio de la investigación y tomando las medidas pertinentes (amonestación, sanción) o por el contrario no se toma en cuenta y la situación no es sancionada como es debida. Si no se adoptan las medidas oportunas desencadenan la cuarta fase.

Fase 4: Exclusión de la vida laboral. En esta fase el trabajador presenta un deterioro de su estado anímico lo cual se traduce en largas temporadas de baja médica.

En las organizaciones privadas el trabajador decide abandonar el trabajo, en la Administración pública podría elegir un cambio de destino (forzoso). En 
cuanto a la tipología del acoso laboral, según Abajo (2004), se distinguen diferentes manifestaciones:

- Mobbing horizontal: se da entre trabajadores de misma escala jerárquica. Para Leyman (1996), las causas de este tipo de acoso se resumen en un grupo de trabajadores fuerza a otro a conformarse con las normas fijadas por la mayoría, enemistad personal, falta de trabajo o aburrimiento o discriminación hacia la víctima por presentar alguna diferencia.

- Mobbing descendente: Quien ostenta el poder acosa a uno o varios trabajadores situados en un nivel inferior dentro de la escala jerárquica.

- Mobbing ascendente: Consiste en aquella en la que una determinada persona que ocupa un cierto grado jerárquico de tipo superior dentro de la organización es acosada por uno o varios de sus subordinados.

- Mobbing mixto: se trata de un tipo de acoso que conjuga el vertical descendente y horizontal. Se produce con motivo de que la jerarquía superior no tome medidas al respecto o permita que el acoso laboral continúe a sabiendas de éste (Escudero y Poyatos, 2004).

De estos tipos de acoso, el más grave es el descendente puesto que supone una condición de inferioridad en la víctima, la cual se encuentra muy limitada para poder reaccionar.

Tras lo anteriormente mencionado, en el ámbito de las Administraciones públicas los conflictos que se suelen dar identificados por Ramió (1996), son: a) conflictos entre los puestos de naturaleza política y los de naturaleza profesional, b) conflictos entre los funcionarios que pertenecen al cuerpo general y los funcionarios que pertenecen a cuerpos especiales, c) conflictos entre los diferentes modelos profesionales de la administración, expresados por grupos (A, B, C, D y E).

\section{El conflicto en la Administración Pública: su determinación y efectos}

En el sector privado es muy frecuente terminar pronto con el problema objeto de conflicto, sin embargo en el sector público se intenta la sanción e incluso el traslado forzoso de la víctima².

2 Libro Blanco «Los riesgos psicosociales en la Administración». Resultados del Barómetro Cisneros $\mathrm{V}$ sobre violencia en el entorno laboral en las Administraciones públicas. El Barómetro Cisneros es un cuestionario desarrollado por el profesor Ińaki Pińuel, para sondear el acoso laboral en las organizaciones. Se compone de 43 ítems que objetivan y valoran conductas. Mirar también Informe UMIST de la Universidad de Manchester, estudio llevado a cabo en una muestra de 5.288 personas de organizaciones profesionales diferentes. Disponible en: http://www.adapttech.it/old/files/ document/19764Destructiveconfl.pdf. 
El conflicto como apunta Quera (2003:125) tiene su origen en actos de violencia, presión, abusos de poder.

El acoso laboral o mobbing evoluciona después de un cierto tiempo de conflicto (Leyman, 1996) a veces, muy rápidamente, otras veces después de semanas o meses. En ese sentido, Arciniega (2012) alude a que en el proceso del mobbing si no se «abre» el conflicto, la víctima no puede defenderse, ni argumentar, ni criticar al acusador. Asimismo, Einarsen (1999) considera que en los ambientes donde no se maneja constructivamente los conflictos se puede intensificar la frustración y los desacuerdos, generando esto problemas interpersonales dentro de la organización, donde el objetivo último para las partes implicadas es machacar y destruir al oponente. Según Luna (2003: 45)

En los casos en que existe una relación de poder entre los que intervienen en el proceso, incluso en los que ocupan categorías laborales idénticas, ocurren situaciones en las que se otorga más poder a una parte que a otra por motivos de antigüedad, vínculos con personas cuyo poder es aún mayor, o ubicaciones en departamentos con algún peso específico.

Escudero (2005) apunta a que este tipo de hostigamiento donde se produce con más frecuencia es en los centros del Sector Público, tal y como menciona la Asociación Española contra el Acoso Psicológico, especialmente en los sectores educativo y sanitario. Actualmente muchas de estas organizaciones no cuentan con mecanismos para afrontar y administrar el proceso. Estudios recientes realizados en los últimos años, afirman que el acoso laboral ha aumentado considerablemente en Europa, más significativamente en países del norte ${ }^{3}$.

Al hablar de Administraciones públicas, Molina (2001: 2) considera el acoso:

Como el resultado de las leyes de inercia histórica y la obsolescencia de buena parte de sus paradigmas de regulación organizativa, predominan las estructuras cerradas, el reglamentismo y una cultura de gestión que sigue considerando el poder y el control como valores prioritarios para conseguir la eficacia, frente a otros parámetros propios de las organizaciones de empresa, como la productividad, la eficiencia, la competitividad o, incluso, la racionalidad.

De igual forma Rivas (2005) sostiene que entre las posibles causas podemos encontrarnos, la estabilidad funcionarial, su régimen de promoción y ascensos y ocupación de determinados cargos de responsabilidad, como las posibles causas explicativas del acoso en la función pública. Seguidamente Einarsen y Hauge

\footnotetext{
${ }^{3}$ Informe Eurofound: «La violencia y el acoso en el lugar de trabajo en Europa: alcance, repercusiones y políticas en el lugar de trabajo en Europa , del 27/02/2015, disponible en: https://www. eurofound.europa.eu/sites/default/files/ef_publication/field_ef_document/ef1473es1.pdf
} 
(2006: 255) mencionan como posibles causas la aparición de desequilibrios de poder.

Lo anteriormente expuesto hace alusión a las causas que desencadenan el acoso laboral en la función pública, no hay que olvidar que en el Estatuto Básico del Empleado público se tipifica tal conducta como falta disciplinaria muy grave (art. 95$)^{4}$, a su vez en el en el art. $14^{5}$ del citado Estatuto se menciona el respeto a la intimidad, y que dicha conducta se produzca dentro de la relación laboral.

Así pues se plantean una serie de interrogantes sobre ¿qué conductas serían calificadas como constitutivas de acoso laboral? y ¿cuáles serían sus efectos? Atendiendo a lo dispuesto en el protocolo de actuación del acoso laboral de la Administración General de Estado ${ }^{6}$ se recogen una serie de conductas que se consideran acoso laboral:

- Dejar al trabajador sin ocupación efectiva durante un tiempo, dejarlo incomunicado, sin ninguna causa que lo justifique.

- Dictar órdenes de obligado cumplimiento con los medios que al trabajador se le asignan, ocupar al trabajador con tareas inútiles o que no tienen valor productivo.

- Llevar a cabo acciones de represalia frente a trabajadores que han planteado quejas, denuncias o demandas frente a la organización, o frente a los que han colaborado con los reclamantes. Insultar o menospreciar repetidamente a un trabajador.

- Reprenderlo reiteradamente delante de otras personas.

— Difundir rumores falsos sobre su trabajo o vida privada.

Tras hablar de los motivos, resulta indispensable el conocer los efectos que el acoso laboral produce, no sólo en la figura del trabajador, sino también en la propia organización. Así pues, a nivel psicológico Leyman (1996: 168) afirma que las repercusiones más habituales están relacionadas con estados de ansiedad que pueden generalizarse a otras situaciones de la vida cotidiana. En este sentido, los efectos que produce una situación de acoso en el trabajador pueden ser

${ }^{4}$ Estatuto básico del empleado público, art. 95.2.b) falta disciplinaria muy grave, «toda actuación que suponga discriminación por origen racial o étnico, religión o convicciones, discapacidad, edad $u$ orientación sexual, lengua, opinión, lugar de nacimiento o vecindad, sexo o cualquier otra condición o circunstancia personal o social, así como el acoso por razón de origen racial o étnico, religión o convicciones, discapacidad, edad u orientación sexual y el acoso moral, sexual, y por razón de sexo".

5 Art. 14. Derechos individuales, h) respeto a su intimidad, orientación sexual, propia imagen y dignidad en el trabajo, especialmente frente al acoso sexual y por razón de sexo, moral o laboral.

${ }^{6}$ Protocolo de actuación frente a la violencia en el trabajo en la Administración general del Estado y los organismos públicos vinculados o dependientes de ella. Resolución de 26 de noviembre de 2015, de la Secretaría de Estado de las Administraciones públicas, por la que se aprueba dicho protocolo. Disponible en: https://www.boe.es/boe/dias/2015/12/10/pdfs/BOE-A-2015-13388.pdf 
el desencadenante de una baja médica. En relación a las consecuencias en la organización, se produce un incremento en la rotación y por ende una disminución de la productividad.

Con respecto a la rotación, actualmente en las investigaciones existentes sobre el acoso laboral, existen estudios que muestran, que la rotación en el trabajo es una consecuencia, por el sentimiento de abandono hacia el puesto de trabajo que muestra la víctima (Hoel y Cooper, 2000: 5) por el contrario, Tepper (2000: 183) pone de manifiesto que las dificultades de encontrar un nuevo empleo debido a la inestabilidad del mercado laboral, evitan que muchas víctimas de acoso teman abandonar su trabajo. El resultado es un empeoramiento de la situación, que lleva a los trabajadores a una situación improductiva.

Como se observa, se pone de manifiesto, que resulta necesario atender y por tanto, poder dar respuesta a esas cuestiones que suscitan inseguridad en el trabajador ocasionando un ambiente hostil. A día de hoy, sigue siendo un problema real, que no sólo incide en la salud del propio trabajador, sino que incide directamente en los resultados de la propia organización. Es necesario poner en práctica instrumentos, dotar al trabajador de técnicas y herramientas, que no sólo regulen sino que también lo protejan, para poder luchar así contra la lacra del acoso laboral.

\section{La mediación como herramienta para la resolución de conflictos en el sector público. Especial referencia a protocolos de actuación y prevención}

Existen diversas formas para prevenir las conductas de acoso, en el caso que nos ocupa se menciona la mediación, como método alternativo de resolución de conflictos.

De la mediación se puede decir que tiene una larga historia. Desde siempre han existido fórmulas de mediación con la intervención de un tercero neutral para dirimir los conflictos. En España, tras la aprobación de la Ley 5/2012 de Mediación de Asuntos Civiles y Mercantiles, se ha hecho hincapié en el uso de la misma. Fruto de la notable relevancia que ha ido cobrando la mediación, al ser extrapolada a diversos ámbitos, surgen numerosas definiciones.

En concreto, Haynes (1995: 11) define la mediación como un proceso en el cual una tercera persona ayuda a los participantes a manejar el conflicto. A la definición dada por Haynes se suma la establecida por Folberg y Taylor (1996: 27) para quienes la mediación:

Es aquel proceso en el que los participantes junto con la asistencia de una persona o personas neutrales aíslan sistemáticamente los problemas en disputa con el ob- 
jeto de encontrar opciones, considerar alternativas y llegar a un acuerdo mutuo que se ajuste a sus necesidades.

Concepto que es completado por Moore (1995: 44) quien subraya que:

La mediación es la intervención en una disputa, de un tercero imparcial y neutral que carece de poder de decisión para ayudar a las partes en disputa a alcanzar voluntariamente su propio acuerdo mutuamente aceptable.

En resumen, estas tres definiciones comparten que la figura de un tercero es clave en un proceso de mediación y que la imparcialidad y la neutralidad son dos características esenciales en todo proceso de mediación. En cuanto a la neutralidad para Guillén y de Diego (2010: 71)

La neutralidad es una característica básica, el mediador es un tercero neutral, el que ejercita la neutralidad desde la participación y el compromiso, el que ejerce una autoridad equilibrada, poniéndose al servicio de las partes.

Así pues, tal y como sostiene Novel (2010: 72) el mediador no forma parte de los intereses de la organización, ni forma parte de las partes en conflicto, ni tiene intereses en ello. Al hablar de mecanismos de adopción de medidas de actuación y prevención adoptadas por organismos públicos nos encontramos con que en muchas ocasiones el mediador es designado por el Comité de Seguridad y Salud, es decir por la propia organización. Ante tal cuestión, es necesario preguntarse si al pertenecer a la propia organización el mediador sería todo lo neutral que tiene que ser o si por el contrario el hecho que sea externo a la organización, le llevaría afrontar el caso con más neutralidad e imparcialidad.

A continuación se plantean una serie de protocolos establecidos por Ayuntamientos en los que se contempla la mediación como herramienta frente situaciones de acoso laboral, y en los que se alude a la figura del mediador.

El protocolo de actuación para la erradicación del acoso laboral establecido por el Ayuntamiento de Alcorcón ${ }^{7}$ (Madrid), se basa en actuaciones que persiguen la investigación, la persecución y la sanción de aquellas conductas que sean calificadas de acoso laboral. En este protocolo se distinguen dos formas distintas de $\operatorname{acoso}^{8}$, por un lado, la denominación de conducta de acoso en la cual están enmarcadas actos y conductas humillantes, vejatorias, intimidatorias, ofensivas,

\footnotetext{
7 Protocolo de prevención y erradicación del acoso laboral en el Ayuntamiento de Alcorcón y sus organismos autónomos: Código Ético de 4/02/2009 disponible en: http://www.solidaridad obrera.org/2009/02/04/protocolo-de-prevenci-erradicaciel-acoso-laboral-en-el-ayto-de-alcorc-ooaaco-ico/

8 Definición de acoso del protocolo de prevención y erradicación del acoso laboral del Ayuntamiento de Alcorcón, en el art.3 se distinguen dos formas distintas de acoso laboral.
} 
etc. Por otro lado, el proceso de acoso, en el cual se engloba las actitudes repetidas o persistentes, perpetradas o por una o más personas, de forma verbal, psicológica o física, en el lugar de trabajo y que tiene por consecuencia la vejación, la degradación, el menosprecio, y las coacciones.

En este protocolo que contempla la mediación, se da la singularidad de que la figura del mediador es designada por el Comité de Seguridad y Salud, el cual se hará cargo de la formación de un grupo de trabajo en prevención de riesgos psicosociales de carácter permanente e interno.

Seguidamente el Ayuntamiento de Casares ${ }^{9}$ (Málaga) en su protocolo de prevención y actuación en caso de acoso también contempla que el caso sea tratado a través de la mediación, considerando la figura del mediador. El mismo será designado por el Comité de Seguridad y Salud, pero apunta a que deberá ser una persona distinta a las que componen el Comité de Seguridad y Salud, garantizando así aún más la imparcialidad y la confidencialidad del proceso de mediación, siendo estas características intrínsecas del proceso de mediación.

Por su parte, el protocolo de actuación frente al acoso del Ayuntamiento de Granada ${ }^{10}$, además de incluir la mediación, propone la formación en mediación mediante un curso de especialización en habilidades sociales y personales, incluyendo la mediación, el control de estrés, o la resolución de conflictos. En caso de denuncia de acoso laboral la misma será investigada por una Comisión de Análisis y Valoración, la cual propondrá el acto de mediación, que será llevado a cabo por un miembro de los designados por el Comité de Seguridad y Salud laboral para formar parte de la Comisión de Análisis y Valoración, distinto de los titulares de ésta.

En el caso del Ayuntamiento de Villanueva de Segura (Murcia) ${ }^{11}$ en su protocolo de actuación en caso de acoso laboral se desprende que si se trata de una situación de conflicto, se propone la actuación de un mediador, admitido por las partes, pero nada dice al respecto sobre el nombramiento del mediador, si tiene que formar parte de la organización o si tiene que ser un mediador externo.

Como se evidencia, en estos protocolos figura la mediación como vía para la resolución del conflicto, pero nada dicen al respecto de la formación en me-

9 Protocolo de prevención y actuación en los casos de acoso laboral, sexual y por razón de sexo u otra discriminación, en el Excmo. Ayuntamiento de Casares (Málaga) disponible en: http://www. casares.es/export/sites/default/MUNICIPIOS/29041/es/archivos/ficheros/arc_20161110_66590.pdf

10 Protocolo de prevención y actuación ante posibles situaciones de acoso laboral, acoso sexual, acoso por razón de sexo ante cualquier discriminación en el ámbito laboral en el Ayuntamiento de Granada, disponible en: http://www.granada.org/inet/wordenanz.nsf/93953ad78e19e38ec1257355 00246d99/4f9dd426a3df8724c1257f53002e3b95!OpenDocument

11 Protocolo de actuación frente al acoso en el entorno laboral del Ayuntamiento de Villanueva del río Segura (Murcia) disponible en: http://www.villanuevadelsegura.es/plan-contra-el-acosolaboral-15/Protocolo_contra_el_acoso_laboral_Villanueva-del-Rio-Segura.pdf 
diación y en técnicas para la resolución de los conflictos, sólo un protocolo de los mencionados esboza la idea de la formación en gestión de conflictos.

\section{Conclusiones}

Este artículo ha tratado de mostrar la importancia de considerar los factores organizacionales en la constitución y el desarrollo del acoso laboral, en particular, la articulación de una serie de características que definen la gestión del personal de la Administración pública.

Son muchas las definiciones que existen de acoso laboral desde diferentes ámbitos, aquí se ha mencionado el ámbito de la psicología, el derecho, la sociología, que llevan a destacar que el acoso laboral tiene como singularidad la actitud de hostigar y la persistencia de las conductas, que dan como resultado la situación de acoso.

En cuanto a los conflictos que se dan en el seno de las Administraciones públicas caben destacar los conflictos interpersonales y los conflictos estructurales, y la distinción en cuanto a la gestión de personal de las organizaciones privadas que cada vez más, se están adaptando al nuevo mercado laboral, caracterizado por una alta competitividad, lo que hace que los recursos humanos sean el valor más importante de la organización. Por el contrario, en las Administraciones públicas falta esa cultura de gestión basada en los recursos humanos y en la adopción de políticas que mejoren las capacidades de su personal. Hace falta un replanteamiento de roles, y una mayor participación del trabajador público.

Cabe reseñar la importancia de la formación del personal. De los protocolos, mencionados anteriormente sólo uno contempla la formación en resolución de conflictos, y ninguno contempla que el mediador sea externo a la organización, por lo que la neutralidad e imparcialidad del mediador en el caso puede verse amenazada. La falta de un modelo consensuado entre los diferentes protocolos mencionados, en lo que se refiere a la figura del mediador, ya que aun contemplándolo, muchos hacen alusión a que sea parte del Comité de Seguridad y Salud y al pertenecer a la misma organización pareciera que el principio de neutralidad se viera amenazado. Por lo que la neutralidad, en la figura del mediador plantea todo un desafío.

Sin embargo, el óbice más significativo es la falta de unificación en la figura del mediador y la falta de formación en gestión de conflictos. En muchos casos el mediador formaría parte de la propia organización, lo cual podría verse por los trabajadores como una amenaza, no creyendo en la neutralidad ni en la propia mediación como un método para resolver un conflicto de tal envergadura. 
En definitiva, es necesario la unificación de criterios en cuanto a la adopción de estos protocolos, mayor formación en gestión de conflictos al personal de las Administraciones públicas, investigar en acciones llevadas a cabo a través de la mediación en el tema que nos ocupa, para poder detectar tanto fortalezas como debilidades. Es preciso determinar qué experiencias hay en este campo, y poner el acento en seguir avanzado y en comprobar si la mediación es un método efectivo para tratar el acoso laboral en el ámbito de las Administraciones públicas.

\section{Bibliografía}

Abajo Olivares, Francisco Javier (2006): Mobbing. Acoso psicológico en el ámbito laboral, Buenos Aires, Lexis Nexis.

Arciniega Arce, Rosa Silvia (2009): «El Acoso Moral (Mobbing) en las Organizaciones Laborales». Revista Psicología Iberoamericana, Vol.17, núm.2, 13-23.

Einarsen, Stale y Hauge, Lars (2006): "Antecedentes y consecuencias del acoso psicológico en el trabajo: una revisión de la literatura», Revista de Psicología del Trabajo y de las Organizaciones, vol. 22(3), 251-273.

Escudero, José Francisco y Poyatos, Gloria (2004): Mobbing: Análisis multidisciplinar y estrategia legal, Barcelona, Bosch.

Folberg, Jay y Taylor, Adam (1996): Mediación: resolución de conflictos sin litigio, México, Limusa Noriega Editors.

García Sánchez, Isabel María (2007): «La Nueva Gestión Pública: evolución y tendencias». Revista Presupuesto y gasto público. Instituto de Estudios Fiscales, 47, 37-64.

Goiría Ormázabal, Juan Ignacio, Olaizola Nogales, Iñaki, San Sebastián, Javier (2007): «La dimensión preventiva: evaluación y planificación de los riesgos psicosociales en las administraciones públicas», en Marcos González, Juan Ignacio y Molina Navarrete, Cristóbal (coords.), El Mobbing en las Administraciones públicas: cómo prevenirlo y sancionarlo. Navarra, Thomson Aranzadi.

GonzÁlez Navarro, Francisco (2009): La dignidad del hombre y el acoso psíquico en el trabajo que se presta en una Administración pública, Pamplona. Thomson Reuters. Aranzadi.

Guillén Gestoso, Carlos y De Diego Vallejo, Raúl (2010): Mediación: Proceso, tácticas y técnicas, Madrid, Pirámide.

Haynes, John (1995): Fundamentos de la mediación familiar: manual práctico para mediadores, Madrid, Gaia.

Hoes, Helge y Cooper, Cary (2000): Destructive conflict and bullying at work. Manchester School of Management, University of Manchester, UK, Institute Science and Technology.

Leymann, Heinz (1996): "Contenido y Desarrollo del Acoso Grupal Moral (Mobbing) en el Trabajo». European Journal of Work and Organizational Psychology, Vol. 5, núm. 2, 165-184.

Luna Lozano, Manuel (2003): Acoso Psicológico en el Trabajo (Mobbing), Madrid, Editorial GPS.

Molina Navarrete, Cristóbal (2001): «La tutela frente a la violencia moral en los lugares de trabajo: entre prevención e indemnización", Revista de Aranzadi Social, núm. 18. 
Molina Navarrete, Cristóbal (2006): «La tutela frente al acoso moral en el ámbito de la función pública: cómo vencer las persistentes resistencias» en Correa Carrasco, Manuel, Acoso Moral en el Trabajo. Concepto, prevención, tutela procesal y reparación de daños, Navarra, Thomson-Aranzadi, 235-271.

Moore, Christopher (1995): El proceso de mediación. Métodos prácticos para la resolución de conflictos, Barcelona, Ediciones Granica.

Muñoz Belmar, Alejandro (2003): «El juego de rol recurso metodológico para la resolución de conflictos escolares» en Vinyamata, Eduard. Aprender del conflicto: conflictología y educación, Barcelona, Graó, 95-110.

Novel Martí, Gloria (2010): Mediación Organizacional: Desarrollando un modelo de éxito compartido. Instituto complutense de mediación y gestión de conflictos, Madrid, Editorial Reus.

PIÑuel, Iñaki (2007): El mobbing o acoso psicológico en el trabajo, en Mir Puig, Carlos (dir), El mobbing desde la perspectiva social, penal y administrativa, Madrid, Consejo General del Poder Judicial, 15-83.

Piñuel, Ińaki y Oñate, Araceli (2004): Informe Cisneros: libro Blanco, Riesgos Psicosociales en la administración: La incidencia del mobbing y el Burnout en la AEAT y La IGAE, Madrid, Sindicato Gestha.

Quera Colomina, Pilar (2003): «El conflicto; escuchar, aprender y crear», en Vinyamata, Eduard. (ed.), Aprender del conflicto. Conflictología y educación, Barcelona, Graó, 123142.

Ramió Matas, Carlos (1999): Teoría de la Organización y de la Administración Pública, Madrid, Tecnos.

RAYNer, Charlotte y CoOper, Cary (1997): «Workplace bullying: myth or reality can we afford to ignore it». Leadership and Organization development Journal, 18, 211-214.

Rogers, Kimberly y Chapell, Duncan (2004): «Prevención y respuesta ante la violencia en el trabajo». Informes OIT, núm.68, Ministerio de Trabajo y Asuntos Sociales.

Tepper, Bennett (2000): "Consequences of abusive supervisión». Academy of Management Journal, 43(2), 178-190.

TouzArd, Hubert (1981): La mediación y la solución de conflictos, Barcelona, Herder.

Vinyamata Camp, Eduard (2003): Aprender del conflicto: conflictología y educación, Barcelona, Graó. 